\title{
Physiologically based mathematical modelling of solute transport within the epidermis and dermis
}

\author{
Joshua J. Calcutt ${ }^{1}$, Yuri G. Anissimov ${ }^{1,2}$ \\ ${ }^{1}$ School of Environment and Science, Griffith University, Gold Coast, Queensland, 4222, \\ Australia \\ ${ }^{2}$ Institute of Molecular Medicine, Sechenov First Moscow State Medical University, Moscow, \\ Russia
}

\begin{abstract}
The stratum corneum is the main barrier to transdermal drug delivery which has previously resulted in mathematical modelling of solute transport in the skin being primarily directed at this skin layer. However, for topical treatment and skin toxicity studies, the concentration in the epidermis and dermis is important and needs to be modelled mathematically. Hitherto, mathematical models for viable skin layers typically simplified the clearance of solute by blood, either assuming sink condition at the top of the skin capillary loops or assuming a distributed clearance in the dermis. This paper is an attempt to develop a physiologically based mathematical model of drug transport in the viable skin. It incorporates explicit modelling of the capillary loops within the dermis and employs COMSOL Multiphysics ${ }^{\circledR}$ software to model the transport in three dimensions. Previously derived simplified models were compared to the results from this new numerical model. The results of this comparison showed that the simplified model reasonably described the average concentration in the viable skin layers when parameters of the models were chosen appropriately. When the recruitment of the capillary loops in the dermis was full and the top of capillary loops was at a depth of $100 \mu m$, the effective depth to place a sink condition in the simpler models was found to be at $150 \mu \mathrm{m}$. However, when there was only partial recruitment of the
\end{abstract}


capillaries, the effective depth increased to $180 \mu \mathrm{m}$. The presented modelling is also essential for determining a transdermal flux when the stratum corneum barrier is compromised by such methods as microporation, application of chemical enhancers or microneedles.

Keywords: Dermal Transport, Diffusion, Capillary Clearance, COMSOL ${ }^{\circledR}$ 


\section{Introduction}

The skin is the largest organ in the body and lowers an organisms exposure to environmental toxins and water evaporation loss. The outermost layer of the skin, the stratum corneum, is the main barrier to transdermal drug delivery. The stratum corneum is composed of a dense set of corneocytes and lipids (similar to a brick and mortar) that prevent penetration of many external substances.[1-5] For this reason, most mathematical models investigate the rate-limiting barrier of the stratum corneum. [6-8] However, for topical dermal drug delivery, where achieving local therapeutic effect is necessary, predicting concentration in the viable epidermis and dermis is important. The application of cosmeceutical products also requires an accurate prediction of the concentration in deeper layers of the skin as toxicity may arise. Similarly, many skin problems such as chloracne and quartz induced scleroderma can arise from excessive exposure to different environmental compounds and chemicals. $[9,10]$ The concentration of the compounds/chemicals in the dermis governs the severity of each of these diseases, so it is important to identify how each of these chemicals will permeate the deeper skin layers when they come into contact with the skin.

The permeability and concentration within the viable epidermis and dermis also become far more consequential when investigating transdermal drug delivery of solutes with very high lipophilicity $(\log P \geq 3$ ), low melting point, low molecular mass and adequate solubility in water and oil. [11, 12] When these conditions are satisfied, the hydrophilic viable epidermis layer presents a significant barrier to highly lipophilic solutes. $[13,14]$ When the stratum corneum barrier is decreased or bypassed through different permeability enhancement techniques (such as iontophoresis, chemical enhancers and microporation), the viable skin can also become the main barrier to transdermal drug delivery. When the viable skin becomes the rate-limiting factor, the transport within the viable epidermis and 
dermis becomes far more important. $[15,16]$

While numerous mathematical models have been developed to express drug transport in the stratum corneum, far less have been formulated to predict transport in the viable skin. In previous models of dermal solute transport, it was assumed that continuous blood clearance happens throughout the tissue. $[17,18]$ In these models, since there is constant blood clearance, a distributed elimination model was introduced to describe how the concentration of a solute decreases with depth in the viable skin. In [17], the concentration depth profile of a distributed elimination model was compared to various experimental data sets to show the validity of the approach. A similar distributed elimination model was utilised in $[19,20]$ and the latter took into account declining concentration in the donor phase.The distributed elimination model was then expanded upon in [21], where different mathematical models that can predict the toxicity of solutes were described in a review. Within this review, a more advanced two-layer distributed elimination model was introduced to describe the drug transport in the viable epidermis and dermis.

A distributed elimination model was also applied in [22] to compare to human cutaneous data. Within this model, it was demonstrated that highly plasma protein bound drugs exhibited convective blood, lymphatic and interstitial transport which caused the solute to penetrate faster to deeper dermal layers and tissues. However, not all modelling of concentration profiles in the dermis have been done using this distributed equation. One of the first attempts to model a concentration profile in this region of the skin was performed in $[23,24]$. In these models, a compartmental approach was used to try and simulate the distribution and elimination of salicylic acid in rat skin. A similar compartmental model was used in [25] to describe experimental concentration in rat skin. The applicability of compartmental approaches was discussed in [18] and showed to be consistent with the 
distributed elimination model. More recently, compartments were use to simulate diffusion in the skin and in particular the stratum corneum. [26]

In this work we will investigate the effect capillary loops have on the overall distribution of a solute in the viable skin. A solid foundation for the modelling of capillary loops in the papillary dermis has been previously elaborated in [27-29]. To develop these models, a review into the physiology of the upper papillary dermis was conducted in [30]. In this review, the dermal clearance process was discussed in detail. The physiology obtained in this review was then used to develop a capillarybased model in [27]. The capillary setup that was utilised in this model forms an integral part to our paper. Further expansion upon the capillary arrangement developed in [27] was completed in [28], where the clearance rate of the drugs was further analysed for a number of different compounds in the mammalian dermis. To expand upon this work, the lymphatic vessels and their importance in the skin when modelling dermal drug distribution was discussed in [29]. The transport of higher molecular weighted drugs was described more accurately in this model by introducing a lymphatic system into the model. However, our model will vary from previous models as it investigates how various capillary properties and variables will affect the overall distribution and transport of a drug.

In this paper, a physiologically based, three-dimensional model was developed to simulate the concentration in the viable epidermal and dermal layers of the skin. Capillary loops in the dermal layer of the skin were explicitly modelled to describe the clearance of solutes by blood. The transport equations were then numerically solved using COMSOL Multiphysics ${ }^{\circledR}$ which applies the finite element method to produce a three-dimensional concentration field. The average concentration at a given depth was later found and compared to a one-layer homogeneous model and a two-layer distributed models to establish their validity.

The three-dimensional model yields the prediction of not only the average con- 
centration but also minimum and maximum concentrations at a given depth, allowing more precise analyses of therapeutic or toxic effects of solutes. Furthermore, this model shows the inaccuracy of developing a model that assumes a sink condition at the bottom of the viable epidermis. Assuming a sink condition at the bottom of the viable epidermis means that the capillary loops are situated directly below this skin layer and that they will eliminate all of the drug in this region. However, this model shows the actual depth a sink condition should be applied to adequately describe viable skin transport and especially concentraion. The effective depth of this sink condition was then shown to change significantly with three physiological variables: the permeability of the capillary wall, the depth of the capillary loop and the distance between each capillary loop.

\section{Method}

\subsection{Formulation of a Mathematical Model}

\subsubsection{Modelling of Capillary Loops}

In this model, a simplistic capillary system will be arranged to simulate the skin. Capillary loops within the skin come from a group of arterial networks (plexuses). $[31,32]$ These capillaries form loops that penetrate through a certain section of the dermis known as the dermal papillae. Each of the capillary loops is separated by some distance as there is one capillary loop present within each dermal papillae cell. [33] The capillary loops then drain into a sub-papillary venular plexus which is a larger venular network that ultimately flows into the larger hypodermal venous network. [34] In this model, the capillaries we will consider will be an extension of the capillary loop in the dermal papillae. By extending these capillary loops, the transport of the solute in deeper layers of the skin will be able to be predicted. However, in future models, where the convective transport of the capillary loops will be considered, the branching of capillary loops and the plexuses that form 
underneath them should be considered.

In order to model the capillary loops, the dimensions of a typical idealised capillary loop (see Fig.1) must be identified. In particular, the height of the loop in the dermis and the width of each loop need to be appropriately estimated. In normal forearm skin, the width of intrapapillary loops was assumed to be $175 \mu \mathrm{m}$ as this was the intermediate value for the width range of $150 \mu m-200 \mu m$ that was found by Umeda et al. [35]. The depth of the top of the capillary loops was set at $100 \mu \mathrm{m}$ as it was assumed that the top of the capillaries would have an average depth that match the depth of the viable epidermis. The diameter of the capillary loop was set to $8 \mu m$ so that it adhered to the typical capillary loop diameter range of $7.5-10 \mu \mathrm{m}$. [36] The distance between each capillary loop was found to be $70 \mu \mathrm{m}$ in previous works through the use of electron microscopy. [37] Finally, the depth of the total computational area was chosen to be $1000 \mu \mathrm{m}$. This depth was chosen as the concentration in this region was found to be infinitesimal when compared to the concentration elsewhere in the computational area. Therefore, it was assumed that any concentration in deeper parts of the dermis would have minimal impact on the results for the transport of a solute. [36] The validity of this claim was later confirmed by using a depth of $1200 \mu \mathrm{m}$ for the computational area and ultimately resulted in an approximate $0.2 \%$ change in the concentration profile. The schematic arrangement of the capillaries and computation area is shown in Figure 1. The shape of the capillaries in this figure were chosen to represent the typical shape of a capillary loop. However, this shape does not consider the possible capillary branching. Future models should investigate the effect such branching would have on the drug transport in the viable skin.

In the model, it is assumed that there is no metabolism of the drug present in the viable skin. However, for some drugs, skin metabolism is a key variable for viable skin and in particular viable epidermal transport. [38] In particular, 

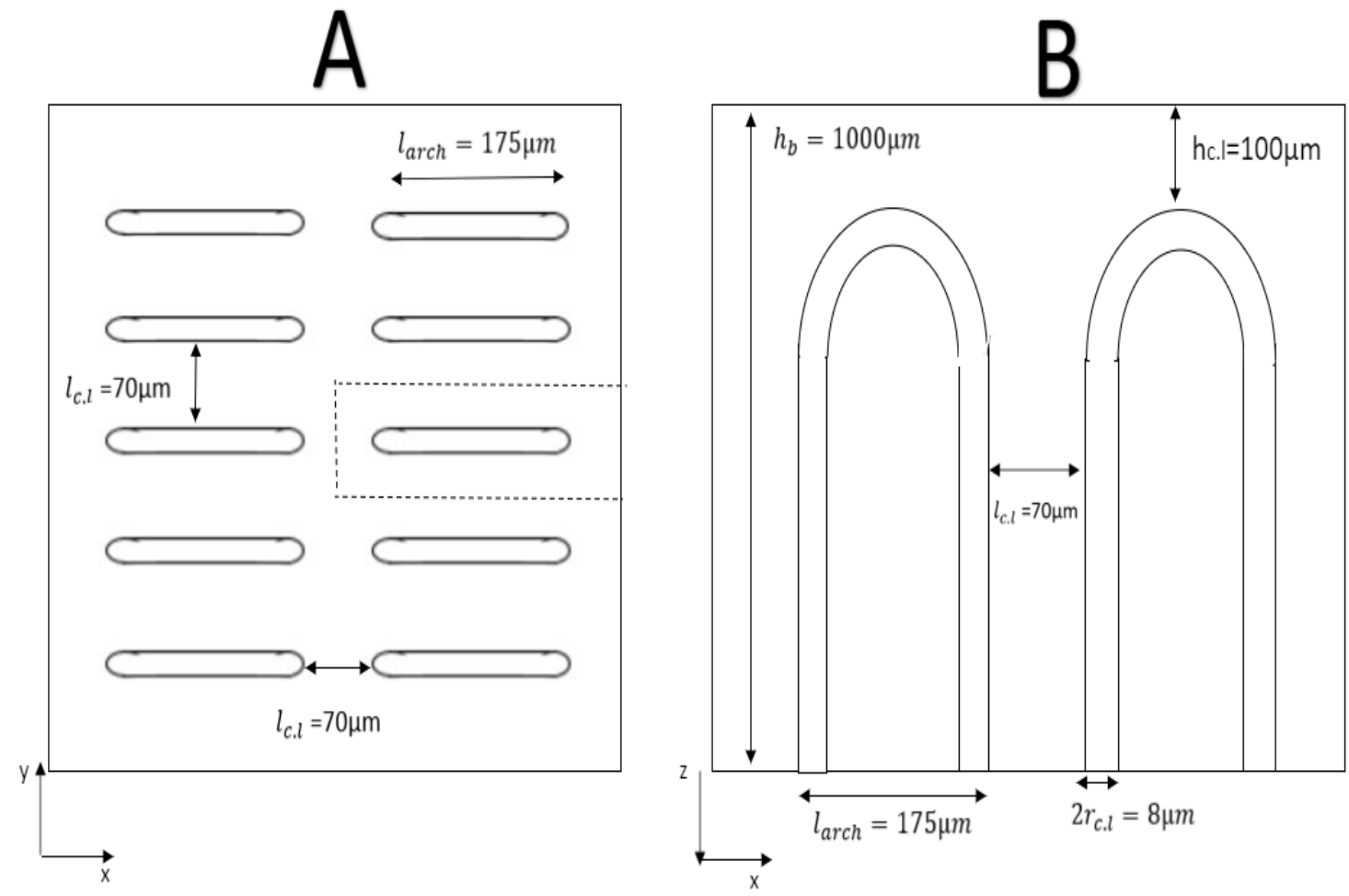

Figure 1: Schematic for the arrangement of idealised capillaries. (A) shows a top view of the capillary loop arrangement while (B) shows a side view. In this diagram, $l_{c . l}$ is the distance between each capillary loop, $l_{\text {arch }}$ is the total width of each capillary loop arch, $r_{c . l}$ is the radius of the capillary loop, $h_{b}$ is the height of the computational area and $h_{c . l}$ is the depth of the capillary loop. Lastly, the dashed lines show a sample computational area that only has one capillary loop.

presence of cytochrome P450's in the differentiating layers of the epidermis and sebacious glands may have some impact on the absorption of some transdermally delivered drug. [39] The use of a diffusion model that incorporates a metabolic process are well discussed in [40] and should be considered further in future models. When there is no metabolism, the solute transport can be described by Fick's second law of diffusion.

$$
\frac{\partial C}{\partial t}=D \nabla^{2} C
$$

where $C$ represents the concentration of solute in the skin, $t$ is time, $D$ is the 
diffusion coefficient of the solute within the skin (which is assumed to be constant across the viable skin) and $\nabla$ represents the vector differential operator del (which is the gradient of the concentration in each coordinate such that $\left.\nabla=\left(\frac{\partial}{\partial x}, \frac{\partial}{\partial y}, \frac{\partial}{\partial z}\right)\right)$. Here $x, y$ and $z$ are the Cartesian coordinates shown in Fig.1. In the viable skin, the diffusion coefficient is often simplified to $D_{v s} \approx 1 / 6 \times D_{a q}$ and this expression has been implemented in this model. [41] The simplification is made as to give a good representation of how many drugs will tranport through the skin when the different physical parameters such as the molecular weight and melting point are difficult to represent.

In order to solve the diffusion equation in (1) boundary conditions must be formulated. The boundary condition used for $z=0$ describes the flux entering the top of the viable skin from the SC:

$$
\left.D \frac{\partial C(x, y, z, t)}{\partial z}\right|_{z=0}=J_{s c}
$$

where $J_{s c}$ is the flux of solute entering the epidermis from the SC.

When there is no metabolism, the capillary loops are the only source of elimination of a solute from the viable skin. Once the drug reaches these capillary loops, the flow of the blood carries the solute along the capillary loop, out of the viable skin and into the systemic circulation. For simplicity, it was assumed that the flow of the blood is quick enough to irreversibly remove all solute that enters the capillary. Therefore, we consider two different boundary conditions for the surface of the capillary loops. The first boundary condition is a sink condition that was assumed on the surface of the capillary loops such that:

$$
\left.C(x, y, z, t)\right|_{S_{C L}}=0
$$

where $S_{C L}$ is the surface of the capillary loops. 
The second boundary condition that was considered is a permeability barrier on the capillary loop wall due to the endothelial lining. However, to consider this boundary condition, one must consider the concentration of the solute inside the capillary loop. When the flow of the capillaries is quick enough to transport to systemic circulation, or there is no chance of repartitioning of the solute once it binds to the red blood cells or blood proteins, the concentration in the capillary can be assumed to be equal to 0 . When there is zero concentration within the capillaries, the following flux condition can be applied to represent the capillary wall permeability barrier.

$$
J_{c l}=-P_{c l} C_{d}
$$

where $P_{c l}$ is the permeability of the solute entering the capillary and $J_{c l}$ is the flux of drug entering the capillary loops.

At the bottom of the viable skin $\left(z=h_{b}\right)$, the concentration of the solute is very small. Therefore, the boundary condition here is assumed to have a minimal effect on the concentration in the rest of the viable skin. Two different boundary conditions were used to test this assumption, a reflective boundary condition such that:

$$
\left.\frac{\partial C(x, y, z, t)}{\partial z}\right|_{z=h_{b}}=0
$$

and a sink boundary condition such that:

$$
\left.C(x, y, z, t)\right|_{z=h_{b}}=0
$$

where $h_{b}$ is the height of the computational area.

On the edges of the computational area, which are shown by the dashed line in Fig. 1A, the flux was set to zero. A zero flux boundary condition is imposed on these edges since the capillary arrangement in this model is assumed to be symmetrical. When there is symmetry of the capillary loops, the following two 
boundary conditions can be applied:

$$
\begin{gathered}
\left.\frac{\partial C(x, y, z, t)}{\partial x}\right|_{x=0, l_{c . a}}=0 \\
\left.\frac{\partial C(x, y, z, t)}{\partial x}\right|_{y=0, w_{c . a}}=0
\end{gathered}
$$

where $l_{c . a}$ is the length of the computational area and $w_{c . a}$ is the width of the computational area.

The numerical solution for this model was obtained through the use of Comsol Multiphysics ${ }^{\circledR}$ software [42]. The results from this model were compared with the simpler one-layer and two-layer models presented below.

\subsubsection{A One-Layer Model}

A one-layer model is a common and simple model that represents skin as a homogeneous membrane. In this model, a one-dimensional form of Fick's second law of diffusion describes the transport of a solute in the viable skin.

$$
\frac{\partial C}{\partial t}=D \frac{\partial^{2} C}{\partial x^{2}}
$$

The boundary condition at the top of the viable skin is the same as in (2). However, since this model cannot incorporate capillary loops to describe the elimination of the solute in the membrane, a sink condition was instead imposed at the bottom of the membrane. When these two boundary conditions were applied, the following expression for the steady-state concentration and flux were obtained:

$$
\begin{gathered}
C_{s s}(x)=\frac{J_{s c} h}{D}\left(1-\frac{x}{h}\right) \\
J_{s s}=\frac{D C_{v}}{h}
\end{gathered}
$$


where $h$ is the depth of the 1-dimensional slab, $C_{s s}$ is the steady state concentration of the solute while $C_{v}=\frac{J_{s c h}}{D}$ and is the concentration of the vehicle at the top of the slab.

\subsubsection{Two Layer Skin Model}

In order to develop a model that was able to predict dermal concentration more accurately, the viable skin was represented as two different regions; the region above the capillary loops and the region below the capillary loops. To simplify these regions, it was assumed that the region above the capillary loops was the viable epidermis and the layer below the capillary loops was the dermis. This assumption is physiologically feasible as the capillary loops form epidermal ridges in the junction between the viable epidermis and dermis. In the viable epidermal layer, there is no elimination of the drug since there are no capillary loops and thus the model is equivalent to the one seen in equation (9). However, in the dermal layer of the skin, an elimination term must be incorporated into the model to represent the elimination of solute from the capillary loops. For this reason, a distributed elimination model was applied to model the concentration of solute in the dermis. [17]

$$
\begin{gathered}
\frac{\partial C_{v e}}{\partial t}=D_{v e} \frac{\partial^{2} C_{v e}}{\partial x^{2}} \\
\frac{\partial C_{d}}{\partial t}=D_{d} \frac{\partial^{2} C_{d}}{\partial x^{2}}-k_{e} C_{d}
\end{gathered}
$$

where $C_{v e}$ is the concentration of solute in the viable epidermis, $C_{d}$ is the concentration of solute in the dermis, $D_{v e}$ is the diffusion coefficient in the viable epidermis, $D_{d}$ is the diffusion coefficient in the dermis and $k_{e}$ is the elimination rate of the solute from the capillary loops.

To solve this system of PDEs, the following boundary conditions were considered. The boundary condition for the flux entering the top of the viable epidermis is the same as in equation (2). Boundary conditions between the viable epidermis 
and dermis represent the continuity of flux and concentration of the solute (the partitioning coefficient between the dermis and viable epidermis was assumed to be one). Finally, at the bottom of the membrane the concentration was assumed to enter systemic circulation and thus vanish as $z \rightarrow \infty$. Laplace tranforms were used to solve equations (12) and (13) together with the boundary conditions where it was assumed for simplicity that $D_{v e}=D_{d}=D$. (see Appendix A for a more detailed solution) The steady-state solution for both layers yields:

$$
\begin{gathered}
C_{v e}^{s s}(x)=\frac{J_{s c}}{\sqrt{D k_{e}}}+\frac{J_{s c} h_{c l}}{D}\left(1-\frac{x}{h_{c l}}\right) \\
C_{d}^{s s}(x)=\frac{J_{s c}}{\sqrt{D k_{e}}} \exp \left(\sqrt{\frac{k_{e}}{D}}\left(h_{c l}-x\right)\right)
\end{gathered}
$$

where $x$ is the depth of the solute in the viable skin.

\subsection{Numerical Simulations}

A numerical solution for the three-dimensional model was obtained using COMSOL Multiphysics ${ }^{\circledR}$. COMSOL ${ }^{\circledR}$ uses the finite element method to solve partial differential equations and is appropriate for solving mass transport problems. When using the finite element method, there is a number of limitations that can cause inaccurate and unrealistic results which were discussed previously by [43]. The form of the concentration field shape and the sensitivity to the position of the boundaries were shown to be the main source of error when using this numerical

method [43]. However, these limitations can generally be overcome through the adequate selection of the shape and size of the mesh.

In this model, a triangular mesh with 100910 elements was used over the computational area. In this mesh, the length of each element in close proximity to the capillary loop was selected to be $2 \mu \mathrm{m}$. The element size was then increased to $10 \mu m$ in regions with less variable concentration (e.g. the edges of the computa- 
tional area).

One last factor that can impact the accuracy of the results is the tolerance. However, COMSOL ${ }^{\circledR}$ has an inbuilt tolerance selector in its software. When completing the simulation, a tolerance of 0.001 was chosen which indicated that the numerical solution was accurate to within $0.1 \%$ at each step.

\section{Results and Discussion}

\subsection{Concentration of a Solute at Different Depths Within the Viable Epidermis and Dermis}

In Figure 2, the maximum, average and minimum concentrations, which were calculated with COMSOL ${ }^{\circledR}$, are compared with the simpler model's theoretical predictions of the average concentration. Above the top of the capillary loops or at depths of down to $70 \mu m$, diffusional transport was the only factor that determined the concentration of the solute. For depths greater than $100 \mu m$, the average concentration of the solute decreased exponentially with depth. The exponential decline present in these layers was found in previous works $[17,18]$ and is due to the capillary loops elimination of the solute.

The sink condition on the surface of these capillary loops also caused a negative exponential trend above the capillary loops (between $70 \mu m-100 \mu m$ ) in the maximum concentration. The impact of the sink condition on the capillary loops was even more pronounced for the minimum concentration as the concentration decreased more rapidly at depths greater than $50 \mu m$. As expected, the minimum concentration decreased to zero in the region of $50 \mu \mathrm{m}$ to $100 \mu \mathrm{m}$ and remained zero thereafter as the sink condition on the loops in this region ensured a zero minimum concentration.

Figure 2 also compares the two-layered skin model concentration profile(dashdotted line) to the average concentration obtained in the numerical solution. In 


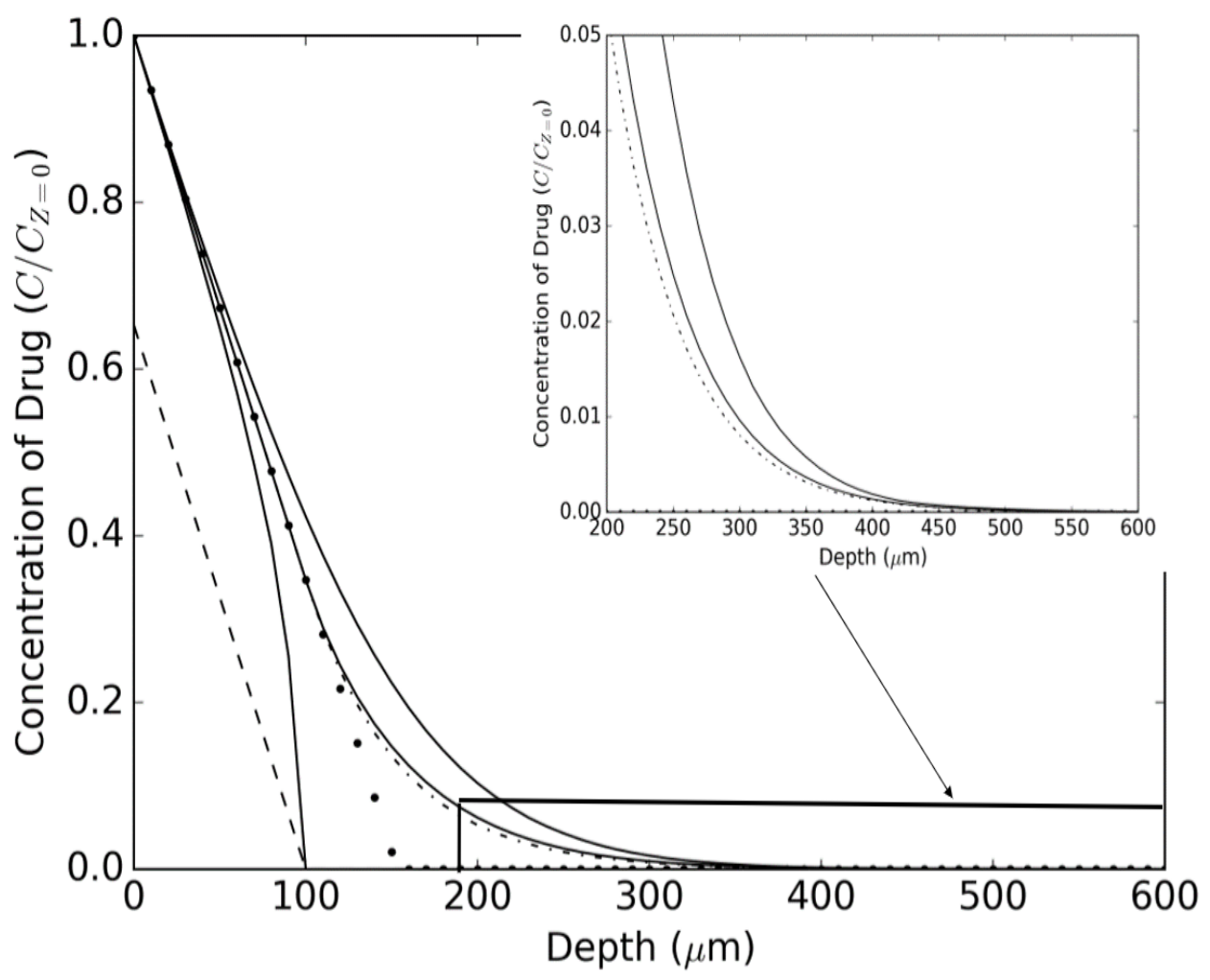

Figure 2: Concentration of a solute at different depths within the viable skin. $C_{Z=0}$ is the concentration at the top of the viable epidermis. In this figure, three solid lines $(-)$ represents the maximum, average and minimum concentration for the numerical solution obtained in COMSOL $^{\circledR}$. The dashed line $(--)$ represents the one layer slab model concentration when using the depth of the capillary loops as the height of the membrane, the dotted line $(\cdots)$ represents the one layer slab model prediction when using the effective depth of the sink condition as the height of the membrane and the dashed and dotted line(-.-) represents the concentration prediction from the two layer slab model.

the viable epidermis layer of the skin (the skin above the top of the capillary loops), the two-layer model predicts the numerical result exceptionally well. It also gives a fair representation of the exponential decline present in the dermal regions of the skin for numerous transdermal drugs. Since the two-layer model effectively shows the concentration profile trend in both layers of the skin, it can be used to give an accurate prediction for the concentration. In order to match 
the two-layer model to the numerical concentration profile, the elimination rate used in the model needs to be adjusted appropriately. The elimination rate of $0.0058 s^{-1}$ was found to replicate the results of the numerical solution in Fig.2. However, various physiological parameters and in particular the distance between each capillary loops can have a significant impact on the elimination rate. For this reason, numerous simulations were used to test the effect each parameter had on the elimination rate and the following expression was obtained:

$$
k_{e}=0.0123-9.3 \times 10^{-5} l_{c . l}
$$

where $l_{c l}$ is in $\mu m$ and $k_{e}$ is in $s^{-1}$.

Here it can be seen that the distance between the capillary loops is inversely proportional to the elimination rate. Other physiological parameters were tested in developing this expression, but were shown to have significantly less impact on the elimination rate and thus were not included.

Figure 2 compares two variations of the one-layer model predictions (dotted and dashed lines) to the average concentration obtained numerically. The first model, which uses the depth of the capillary loops as the height of the membrane (dashed line) has the correct gradient for the concentration in the viable epidermis but underestimates the average concentration throughout this skin layer. The concentration at the top of the viable epidermis for this model was $34.7 \%$ lower than the numerical result. However, using the effective depth of the sink condition as the height of the membrane overcomes this problem. In the particular case where the depth of the capillary loops was set to $100 \mu m$, the effective depth of the sink condition was found to be $153 \mu \mathrm{m}$. Utilising this effective depth estimation in the one-layer model allowed this model's concentration to mirror the trend of the average concentration in the viable epidermis. This is especially the case in 
regions of the viable epidermis closer to the stratum corneum, where the updated one-layer model conforms exceptionally well to the average concentration.

However, the one-layer model has its inherent limitations. Below the top of the capillary loops, it becomes far less accurate and underestimates the numerically calculated concentration. This variance from the numeric solution is especially evident at depths greater than $150 \mu \mathrm{m}$, where the simple model predicts zero concentration. Nevertheless, the one-layer model was able to give an adequate depiction of the concentration of the solute within the viable epidermis and the early dermis while the two-layer model gave a more accurate result throughout the deeper layers of the viable skin.

Figure 3 presents the experimental data for Econazole and the maximum, average and minimum concentration for two different capillary wall scenarios. The first capillary simulation had a sink condition imposed upon the surface of the capillary loop while the second considered a finite permeability of $0.8 \mu \mathrm{m} \mathrm{s}^{-1}$ and a dermis/blood solute partitioning of 4.21.[27] In this figure, it is evident that the average concentration when sink conditions are assumed poorly predicts the experimental concentration of Econazole. One of the main reasons for the inaccuracies of this model is the assumption of a sink condition on the surface of the capillary loops. A sink condition was imposed on the capillary wall to simplify the model, as it was assumed that any solute that came into contact with the capillary loops would permeate through the capillary and into the bloodstream. However when the boundary condition of the capillary loops is changed to incorporate a permeabliity barrier, the numerical result matches the experimental data of Econazole much better.

The endothelial lining on the capillary wall can act as a barrier to solute transport which was not represented in the simplified numerical model since the model assumed a sink condition on the surface of the capillary loop. In order to account 


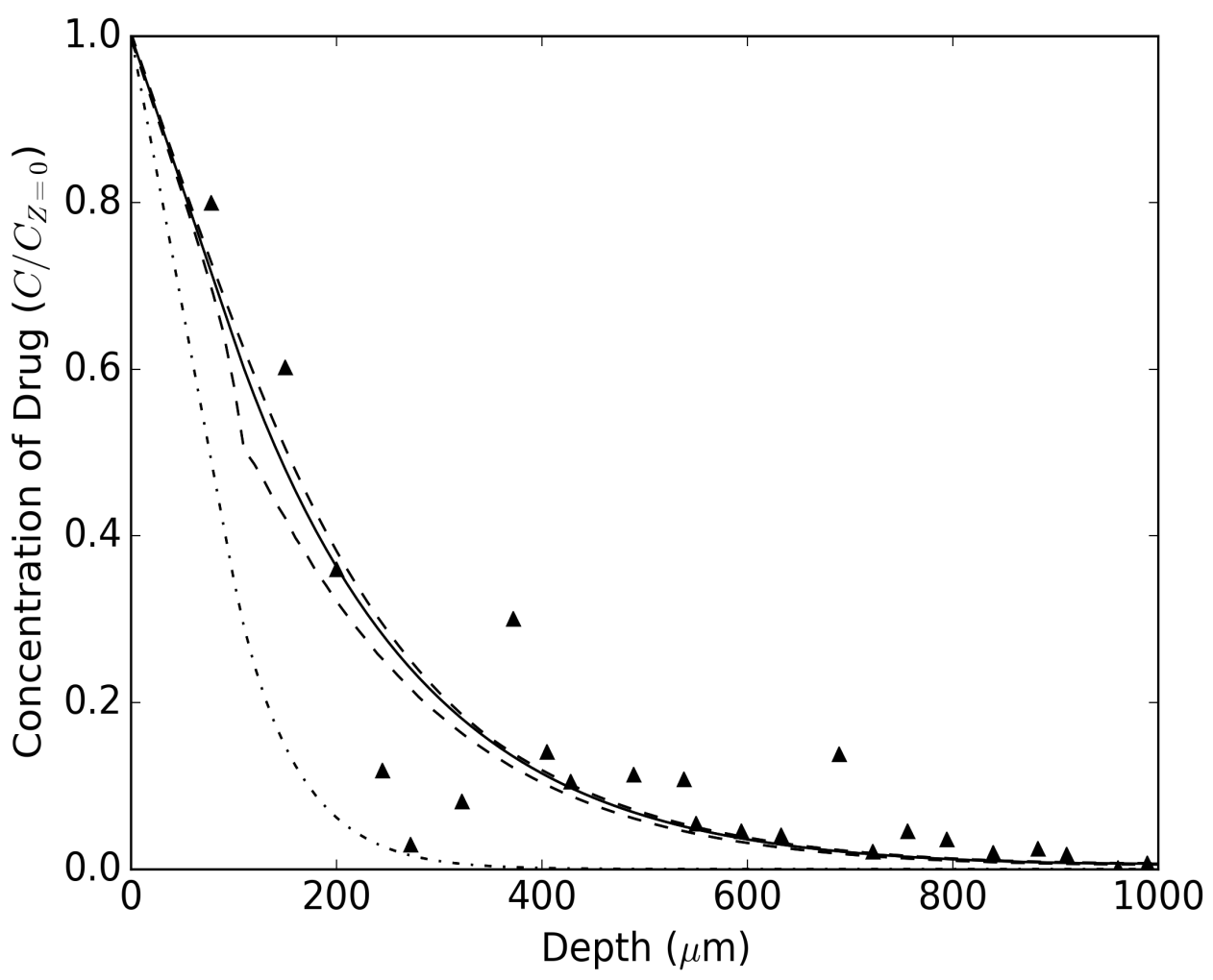

Figure 3: Concentration of a solute at different depths within the viable skin. $C_{Z=0}$ is the concentration at the top of the viable epidermis. In this figure, the dashed-dotted line $(-.-)$ represents the average concentration when using a sink condition, the solid line $(-)$ represents the average concentration for the a capillary permeability of $0.8 \mu \mathrm{m} \mathrm{s} \mathrm{s}^{-1}$. The two dashed lines ($--)$ represent the maximum and minimum concentration when a capillary permeablity of $0.8 \mu \mathrm{m}$ $s^{-1}$ was used while the bolded hats $(\boldsymbol{\Lambda})$ represent the experimental data for the concentration of Econazole in the skin which have been retrieved from [44].

for the permeability barrier of the capillary wall due to the endothelial lining, the numerical simulation was repeated with a permeability of $0.8 \mu \mathrm{ms}^{-1}$. The effects of making this change are evident in Fig.3 where the maximum, average and minimum concentration for both capillary scenarios are compared with Econazole data. The results confirm that the permeability of the capillary wall is a significant factor that needs to be taken into account when modelling the capillary loops. The permeability of the solute also has a significant impact on the effective elimination 
rate parameter $\left(k_{e}\right)$ in the two-layer model. For the permeabiltiy chosen in this model, the effective eliminaiton rate was equal to $5.44 \times 10^{-4} s^{-1}$. The effective elimination rate was seen to drop linearly with the permeability such that:

$$
k_{e}=2.9 \times 10^{-4} P_{c l}+0.00034
$$

where $P_{c l}$ is the permeability of the capillary loop in the dermis. This expression differs from that reported in [17] for the case when penetration of the capillary is the rate limiting factor:

$$
k_{e}=f u_{d} P_{c l}
$$

where $f u_{d}$ is the fraction of unbound drug in the dermis. While the expression obtained in this model differs from the one obtained in [17], the differences can be explained. The model presented in [17] does not consider the physiology of the capillary loops. Also in the present model, the fraction of unbound drug is assumed to be 1 . However, the effect of this fraction of unbound drug was found to be an important parameter in [22] and should be further considered in future models.

However, fitting a permeability value to the experimental data is not without its issues. In order to match the exponential decline in the Econazole dataset, the permeability coefficient mentioned above was chosen for the capillary loop. However, there are discrepancies between the experimental model and the numerical model presented. First of all, the numerical model assumes no repartitioning of solute after it enters the capillary. However, Econazole and other transdermal drugs may permeate through the capillary wall and re-enter the dermal tissue. Repartitioning of the solute will cause an increase in the concentration of solute in deeper dermal tissue and match the trend shown for Econazole much better. If repartitioning was considered in this model (considered in [27]), it could also 
significantly decrease the permeability needed to match the experimental data of Econazole. Another possible factor that may decrease the permeability chosen for this model is the presence of lymphatic vessels throughout the skin. The lymphatic vessels can have a significant impact on dermal transport as they facilitate convective transport in the dermis. The convective nature of the lymphatic vessels will similarly increase the deeper dermal concentration for larger molecular weighted drugs and thus result in a reduction in the permeability of the capillary loop in this model. By taking both of these factors into account in future works, a more detailed physiological model can be obtained.

\subsection{Concentration of the Solute at a Given Depth in the Dermis}

The average concentration-depth profile of a drug is only one of the important parameters that needs to be considered. The location of the maximum and minimum concentration is also an important quantity to consider as it is extremely relevant to toxicologists and pharmacists. For this reason, various depth crosssections were analysed to try and identify the concentration distribution at any depth. Figure 4 gives an example of one of these cross sections by showing the concentration of a solute present at a depth of $300 \mu \mathrm{m}$.

Figure 4A, shows the contour plot of the concentration at a depth of $300 \mu \mathrm{m}$. In this figure, it was evident that the concentration of the solute dropped significantly as the solute approached the surface of the capillary loops. On the other hand, further away from the capillary loops the concentration decreased less rapidly with distance. The stabilisation of the concentration was evident in the figure as the gradients of the contours are much less pronounced between the two capillary loops. The concentration gradients also show that the maximum concentration of the solute will be halfway between the two capillary loops or at approximately $245 \mu m$ on the $x$-axis. The location for this maximum concentration was intuitively expected and was further validated in Figure 4B. 

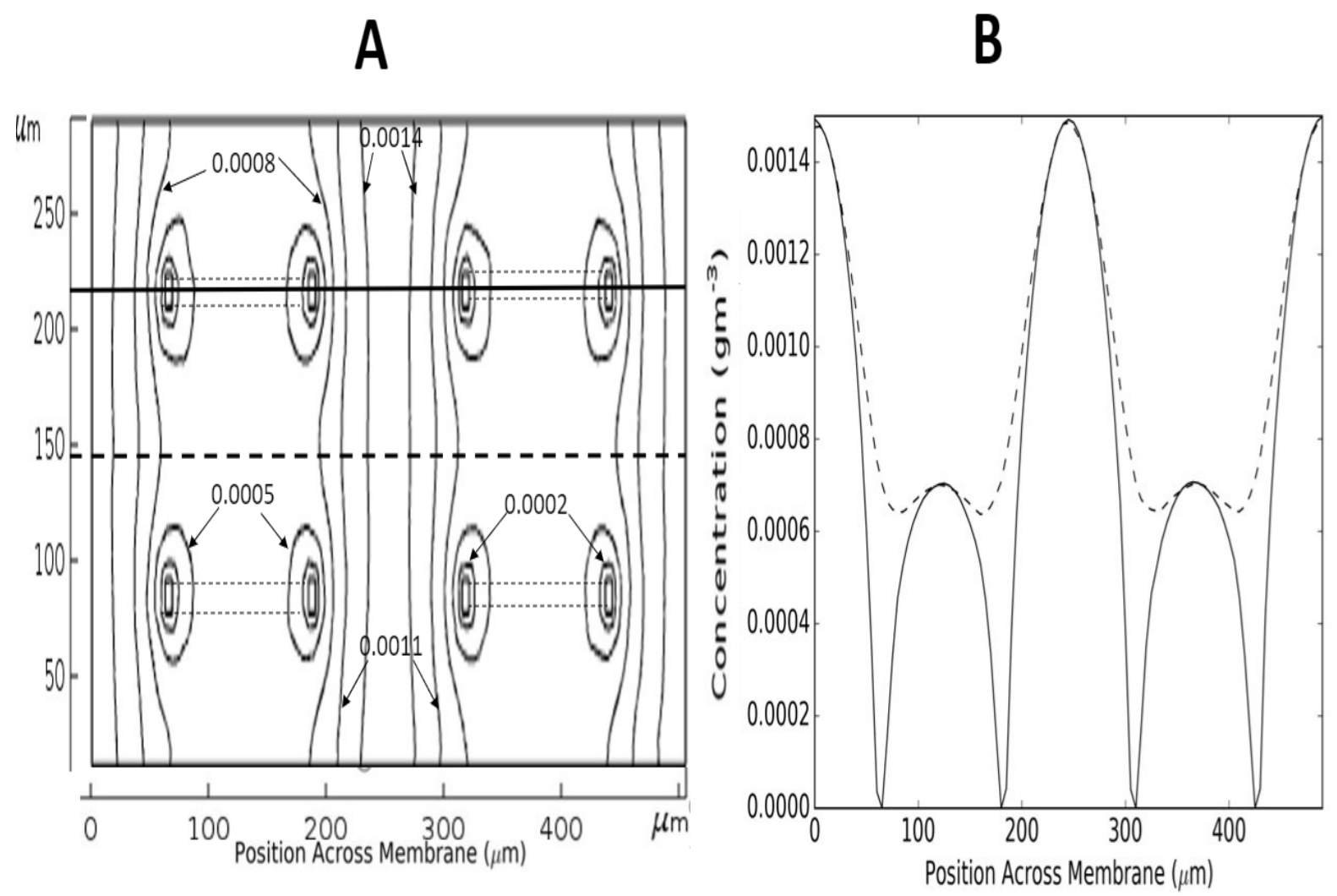

Figure 4: Concentration of a solute at varying positions across the computational area when the depth is set at $300 \mu \mathrm{m}$. Figure A shows a contour plot for the concentration of a solute across this cross-section while Figure B shows how the concentration of the solute changes across two different lines. In Figure B, the dotted line $(---)$ represents the case when the solute is between two capillary loops (as seen by the bold dotted line in Figure A). The solid line represents the case when the solute is in line with a set of capillary loops and occurs when the width on the $\mathrm{y}$-axis of Figure A is equal to $215 \mu m$ (bold solid line).

Figure 4B reciprocates the trends that were evident in Figure 4A but instead shows the concentration across different line cuts of the capillary loop arrangement. In this figure, it is evident from both data sets that the maximum concentration of the solute was found in the middle of the two capillary loops (at $245 \mu \mathrm{m}$ ) or on the edge of the area shown $(0$ and $490 \mu \mathrm{m})$. The maximum values in each of these locations were equal, which is an expected result as the capillary loops were arranged symmetrically. 
When the focus was changed to the cross section in line with the capillary loops (the solid line in Fig.4), the minimum concentration (which is equal to zero) was seen to occur on the capillary loop wall. Again, the zero concentration was an expected result as a sink condition was assumed upon the surface of the capillary loop. When the solute was positioned closer toward the centre of the capillary loop arch, (found at $120 \mu \mathrm{m}$ and $370 \mu \mathrm{m}$ ) the concentration reached a local maximum which was two and a half times smaller than the maximum found in between the capillary loops. Nevertheless, the concentration in this region is still quite large when compared to the rest of the cross-section and thus is essential to consider when investigating the toxicity and therapeutic levels of a solute. However, the likelihood for toxicity in this region is far less significant than that on the solid line on Fig.4B that is in between the two capillary loops.

\subsection{The Effect of Changing the Depth and the Distance Between Each of the Capillary Loop on the Effective Depth of the Sink Condition and the Effective Permeability of a Solute}

From our numerical investigation, it is clear that the capillary loops need to be implicitly investigated to have a detailed concentration profile in the viable skin. However, the exact position and spacing of these loops vary from person to person. For example, in thicker parts of the skin the capillary loops may be deeper within the dermis. The positioning of the loops also depend on physiological conditions, such as temperature, which can change capillary recruitment in the skin. These variables are even more critical when considering the application of microneedles, as the diffusional length the solute has to travel to reach the capillaries decreases significantly.

By using the one-layer model formulated earlier, expressions for both the effective depth of the sink condition and the effective permeability of the solute through the viable skin can be developed. The approach to finding these values is analo- 
gous to the one in [45]. The effective depth of the one-layer model is particularly important as it predicts what depth a sink condition must be positioned to best match the effect the capillary loops have on the transport of the solute (which was seen earlier in Fig.2). To derive a model for the effective depth of the sink condition, equation (11) can be rearranged to obtain:

$$
H_{e f f}=\frac{D C_{v}}{J_{s s}}
$$

where $H_{\text {eff }}$ is the effective depth of the sink condition.

When a sink condition is imposed on the surface of the capillary loops, numerical calculations and linear regressions were used to determine that a linear relationship exists between the effective depth and both the capillary loop's depth $\left(h_{c l}\right)$ and the distance between the capillary loops $\left(l_{c l}\right)$ :

$$
H_{e f f}=0.557 l_{c . l}+0.868 h_{c l}+55.4
$$

Here all the distances are measured in $\mu m$. The accuracy of this expression was tested for numerous capillary loop depths and capillary loop densities, and each scenario showed that this expression was able to accurately estimate (error less than 1\%) the effective depth of the sink condition. Therefore, equation (20) can be used to impose a sink condition at a correct depth for simplified modelling. In particular, this equation can be used in the one-layer model to estimate the depth of a sink condition that should be imposed so that the model can accurately predict the viable skin concentration.

The effective permeability of a drug can be found through the use of the effective depth of the sink condition such that:

$$
P_{e f f}=\frac{D K_{v . s}}{H_{e f f}}
$$


where $K_{v . s}$ is the partitioning coefficient present between the viable epidermis and dermis. From this expression, it is evident that the effective depth of the sink condition is inversely proportional to the effective permeability of a solute. By implementing equation (20), the following expression for the effective permeability was found.

$$
P_{e f f}=\frac{D K_{v . s}}{0.55 l_{c . l}+0.868 h_{c l}+55.5}
$$

where in this equation $D$ is measured in $\mu m^{2} s^{-1}$ and $P_{\text {eff }}$ is measured in $\mu m s^{-1}$.

This equation showed that increasing either the depth or distance between each capillary loops resulted in a decrease in the effective permeability. However, in the case where a sink condition is not assumed on the capillary loops, these effective depths of the sink condition can be modified to incorporate the permeability of the capillary loop wall. In the case where the capillary loop permeability is significantly smaller than the effective permeability of the total skin layer $\left(P_{c l}<<P_{e f f}\right)$, the following expression was obtained for the effective depth of the sink condition.

$$
H_{e f f}=0.98 h_{c l}+1.41 l_{c l}-10.5 P_{c l}+72.31
$$

Here it can be seen that introducing this permeability barrier on the capillary loop wall can significantly increase the effective depth of an assumed sink condition. When a permeability barrier of $1 \mu \mathrm{ms}^{-1}$ is introduced for the capillary loops, the effective depth of the sink condition is seen to increase by $100 \mu m$ to a depth of $258 \mu \mathrm{m}$. Therefore, the capillary permeability has a significant impact on the effective depth of the sink condition and thus should be considered.

\subsection{Sensitivity Analysis of Other Physiological Parameters}

Many other physiological parameters can change the effective depth of the sink condition. The width of the capillaries, diameter of the capillaries, diffusion coefficient in the skin, boundary condition at the bottom of the dermis layer and 
the mesh element size can all alter the transport of a solute in the viable skin. Therefore, the effects of each of these parameters will be investigated in this section.

Width of the Capillary Loops When the width of the capillary loops was increased from $170 \mu m$ to $200 \mu m$, the effective depth of the sink condition decreased by 2 to $3 \mu \mathrm{m}$. On the other hand, when the width of the capillary loops was reduced to $150 \mu m$, the effective depth of the sink condition increased by approximately $2.5 \mu \mathrm{m}$. However, these changes in the effective depth of the sink condition are quite small as they only result in a change of $1.5 \%$ for the effective sink condition depth. Therefore, this variable has limited effect on the effective depth and the concentration of the solute.

Diameter of the Capillary Loops In the body, capillaries are seen to have a variation in both diameter and size. Each capillary will be somewhat unique with a different diameter being present and the possibility for flattening and expansion of the capillaries. For this reason, it is important to consider whether the diameter of these capillary loops has much of an effect on the transport of the solute and the positioning of the sink condition. When the diameter of the capillary loops was doubled from $8 \mu m$ to $16 \mu m$, the effective depth of the sink condition only increased by $2 \mu \mathrm{m}$. However, as seen earlier, the diameter of these capillaries only ranges from $7.5 \mu m$ to $10 \mu m$ [36]. Therefore, this diameter range will only cause a minimal change to both the effective depth of sink position and the transport of the solute.

Diffusion Coefficient From equation (19), it is evident that the diffusion coefficient will not have any effect on the effective depth of the sink condition. However, as a test, simulations were run with different diffusion coefficients. In one of the simulations, the diffusion coefficient was decreased by a factor of 6 . When this change was implemented, the resultant effective depth of the sink remained constant. Therefore, it was evident that the diffusion coefficient did not affect the 
effective depth of the sink condition. Nonetheless, the diffusion coefficient is an important parameter to consider for transient problems.

Boundary Condition on the Bottom of the Computational Area Another parameter to discuss is the boundary condition put at the bottom of the computational area. Initially, it was assumed that there was a reflective boundary condition placed at the bottom of the computational area. However, the physiological relevance of this condition is not apparent. For this reason, a sink condition at the bottom of the computational area was also implemented and compared to the original boundary condition. When a sink condition was used, the concentration of the solute decreased by approximately $0.5 \%$ at depths less than $800 \mu \mathrm{m}$. However, at depths greater than $900 \mu m$, the concentration converges to zero much more quickly than when using a reflective boundary condition. This does not have much of an impact on transdermal delivery as the concentration at this depth is already 10000 times less than the concentration in higher skin layers and therefore is effectively zero in any case. To further investigate the boundary conditions, no boundary condition was specified at the bottom of the computational area. The results of using this boundary condition were seen to conform to the ones seen in the reflective boundary condition case. Therefore, the choice of boundary conditions has a limited impact on the transport of a solute in viable skin.

Mesh Element Size and Shape The mesh element size is the main factor which leads to numerical error. Numerous other mesh combination were investigated to justify the mesh element size used in this model. By increasing the mesh size to a value of 329000 elements, the numerical solution was only seen to increase by $0.3 \%$, and thus it was concluded that the initial mesh selection was adequate. The shape of the elements in the mesh was also changed and had minimal effect on the overall distribution of a solute. 


\section{Conclusions}

In this paper, the effect of explicitly considering capillary loops in the modelling of epidermal and dermal drug transport was investigated using a numerical solution of the transport equation. Previously, capillary clearance was not investigated explicitly and instead was represented by a sink condition or an empirical elimination rate constant.

This work allowed us to represent this rate constant, as well as to establish the correct effective depth of the sink condition as a function of physiological parameters. Assuming a sink condition at the top of the capillary loops underestimates the effective depth of the sink condition by approximately $50 \%$. Furthermore, this error grew when the distance between the capillary loops increased, or the depth of the capillary loops decreased. There was also a significant difference in the maximum and average concentrations at a given depth, and the maximum concentration occurred mid-distance between capillary loops.

An analytically solvable two-layer model was developed and compared to numerical simulations. This model quite accurately agreed with the numerical predictions for the average concentration, given that the value for the elimination rate was appropriately chosen.

In future models of solute transport in viable layers of skin, convective solute transport to deeper skin layers need to be taken into account. Another challenge to future modelling is to investigate how convective flow from the lymphatic vessels affects the concentration of a solute in deeper dermal regions. Furthermore, the metabolism and branching of the capillary loops should also be investigated in future as these two variables may have a significant impact on viable skin transport. Finally, the transient profile of the drug in the viable skin should be investigated in future to see if the diffusion time will change when convective transport is introduced to the model. 
[1] S. Andrews, E. Jeong, M. R. Prausnitz, Transdermal delivery of molecules is limited by full epidermis, not just stratum corneum, Journal of Pharmaceutical Research 30 (4). doi:10.1007/s11095-012-0946-7.

[2] J. Bouwstra, M. Ponec, The skin barrier in healthy and diseased state, Biochimica et Biophysica Acta (BBA) - Biomembranes 1758 (12) (2006) 2080 - 2095. doi:10.1016/j.bbamem.2006.06.021.

[3] S. Chandrasekaran, W. Bayne, J. Shaw, Pharmacokinetics of drug permeation through human skin, J. Pharm. Sci. 67 (10) (1978) 1370-1374. doi:10.1002/ jps. 2600671010.

[4] S. Münch, J. Wohlrab, R. H. H. Neubert, Dermal and transdermal delivery of pharmaceutically relevant macromolecules, Eur. J. Pharm. Biopharm. 119 (2017) 235-242. doi:10.1016/j.ejpb.2017.06.019.

[5] M. Fernandes, L. Simon, N. Loney, Mathematical modeling of transdermal drug-delivery systems: Analysis and applications, J. Membr. Sci. 256 (2005) 184-192. doi:10.1016/j.memsci.2005.02.018.

[6] H. Frasch, A. Barbero, Steady-state flux and lag time in the stratum corneum lipid pathway: Results from finite element models, J. Pharm. Sci. 92 (2003) 21962207. doi:10.1002/jps. 10466.

[7] Y. G. Anissimov, Mathematical models for different exposure conditions in: Dermal Absorption and Toxicity Assessment, no. 271-286, Informa Healthcare, 2008.

[8] Y. N. Kalia, F. Pirot, R. H. Guy, Homogeneous transport in a heterogeneous membrane: Water diffusion across human stratum corneum in vivo, Biophys. J 71 (1996) 26922700. doi:10.1016/s0006-3495(96)79460-2. 
[9] J. English, R. Dawe, J. Ferguson, Environmental effects and skin disease, Br. Med. Bull. 68 (1) (2003) 129-142. arXiv:/oup/backfile/content_ public/journal/bmb/68/1/10.1093/bmb/ldg026/2/ldg026.pdf, doi:10 . 1093/bmb/ldg026.

[10] S. E. Anderson, B. Meade, Potential health effects associated with dermal exposure to occupational chemicals, Environmental Health Insights 8 (2014) 5162. doi:10.4137/ehi.s15258.

[11] S. E. Cross, B. M. Magnusson, G. Winckle, Y. Anissimov, M. S. Roberts, Determination of the effect of lipophilicity on the in vitro permeability and tissue reservoir characteristics of topically applied solutes in human skin layers, J. Invest. Dermatol. 120 (2003) 759-64. doi:https://doi.org/10.1046/ j.1523-1747.2003.12131.x.

[12] H. E. Benson, Transdermal drug delivery: Penetration enhancement techniques, Current Drug Delivery 2 (2005) 23-33. doi:10.2174/ 1567201052772915.

[13] K. McCarley, A. Bunge, Physiologically relevant two-compartment pharmacokinetic models for skin, J. Pharm. Sci. 89 (2000) 1212-35. doi:10.1002/ 1520-6017 (200009)89:9<1212: :aid-jps13>3.3.co;2-w.

[14] A. Bunge, R. Cleek, B. Vecchia, A new method for estimating dermal absorption from chemical exposure. 3. compared with steady-state methods for prediction and data analysis, Pharm. Res. 12 (7) (1995) 972-982. doi: $10.1023 / A: 1016298012408$.

[15] K. S. Paudel, M. Milewski, C. L. Swadley, N. K. Brogden, P. Ghosh, A. L. Stinchcomb, Challenges and opportunities in dermal/transdermal delivery., Therapeutic Delivery 1 (2010) 109131. doi:10.4155/tde.10.16. 
[16] A. Alkilani, M. McCrudden, R. Donnelly, Transdermal drug delivery: Innovative pharmaceutical developments based on disruption of the barrier properties of the stratum corneum, Pharmaceutics 7 (4) (2015) 438470. doi:10.3390/pharmaceutics7040438.

[17] Y. Anissimov, M. S. Roberts, Modelling dermal drug distribution after topical application in human, Pharm. Res. 28 (9) (2011) 2119-2129. doi:10.1007/ s11095-011-0437-2.

[18] K. Kretsos, G. Kasting, J. Nitsche, Distributed diffusion clearance model for transient drug distribution within the skin., J. Pharm. Sci. 93. doi: 10.1002/jps. 20187.

[19] E. Gupta, M. Wientjes, J. Au, Penetration kinetics of 2,3-dideoxyinosine in dermis is described by the distributed model., Pharm Res. 12 (1995) 108-112. doi : $10.1023 / A: 1016298906589$.

[20] S. E. Cross, M. S. Roberts, Defining a model to predict the distribution of topically applied growth factors and other solutesin excisional full-thickness wounds, J. Invest. Dermatol. 112 (1999) 36-41. doi :10.1046/j.1523-1747. 1999.00473.x.

[21] Y. Anissimov, Mathematical models for skin toxicology, Expert Opin. Drug Metab. Toxicol. 10 (4) (2014) 551-560, pMID: 24490982. arXiv:https:// doi.org/10.1517/17425255.2014.882318, doi:10.1517/17425255.2014. 882318.

[22] Y. Dancik, Y. G. Anissimov, O. G. Jepps, M. S. Roberts, Convective transport of highly plasma protein bound drugs facilitates direct penetration into deep tissues after topical application, Br. J. Clin. Pharmacol. 73 (4) (2011) 564-578. arXiv:https://bpspubs.onlinelibrary. 
wiley.com/doi/pdf/10.1111/j.1365-2125.2011.04128.x, doi:10.1111/ j.1365-2125.2011.04128.x.

[23] P. Singh, M. S. Roberts, Blood-flow measurements in skin and underlying tissues by microsphere methodapplication to dermal pharmacokinetics of polar nonelectrolytes, J. Pharm. Sci. 82 (1993) 873-879. doi:10.1002/jps. 2600820903.

[24] P. Singh, M. S. Roberts, Effects of vasoconstriction on dermal pharmacokinetics and local tissue distribution of compounds, J. Pharm. Sci. 83 (1994) 783-791. doi:10.1002/jps.2600830605.

[25] M. B. Reddy, R. J. Looney, M. J. Utell, K. P. Plotzke, M. E. Andersen, Modeling of human dermal absorption of octamethylcyclotetrasiloxane (d4) and decamethylcyclopentasiloxane (d5), Toxicol. Sci. 99 (2) (2007) 422-431. arXiv:/oup/backfile/content_public/journal/toxsci/99/2/ 10.1093_toxsci_kfm174/1/kfm174.pdf, doi:10.1093/toxsci/kfm174.

[26] A. A. Amarah, D. G. Petlin, J. E. Grice, J. Hadgraft, M. S. Roberts, Y. G. Anissimov, Compartmental modeling of skin transport, Eur. J. Pharm. Biopharm. 130 (2018) 336-344. doi:10.1016/j.ejpb.2018.07.015.

[27] K. Krestos, G. B. Kastings, A geometrical model of dermal capillary clearance., Math Biosci 208 (2007) 430-453. doi:https://doi.org/10.1016/j . mbs.2006.10.012.

[28] K. Krestos, M. A. Miller, G. Zamora-Estrada, G. B. Kastings, Partitioning, diffusivity and clearance of skin permeants in mammalian dermis., Int. J. Pharm. 346 (2008) 64-79. doi:10.1016/j.ijpharm.2007.06.020. 
[29] R. Ibrahim, J. M. Nitsche, G. B. Kastings, Dermal clearance model for epidermal bioavailability calculations., J. Pharm. Sci. 101 (2012) 2094-2108. doi:10.1002/jps. 23106.

[30] K. Krestos, G. B. Kastings, Dermal capillary clearance: physiology and modeling., Skin Pharmacol Physiol 18 (2005) 55-74. doi:10.1159/000083706.

[31] W. Spalteholtz, Die verteilung der blutgefabe in der haut, Arch Anat Physiol Anat 2 (1893) 1-54.

[32] E. Landis, The capillaries of the skin: A review, J. Invest. Dermatol. 1 (4) (1938) 295-311.

[33] I. Braverman, Bioengineering of the Skin: Cutaneous Blood Flow and Erythema, CRC Press, 1995.

[34] M. Abrahamson, E. Vazquez-Figueroa, N. Woodall, J. Moore, A. Bommarius, Development of an amine dehydrogenase for synthesis of chiral amines, Angewandte Chemie 51 (16) (2012) 3969-3972. doi:10.1002/anie. 201107813.

[35] N. Umeda, A. Ikeda, Scanning electron microscopic study of the capillary loops in the dermal papillae. skin of the hand of the japanese monkey (macaca fuscata)., Acta Anat. (Basel) 132 (4) (1988) 270-275.

[36] I. Braverman, A. Yen, Ultrastructure of the capillary loops in the dermal papillae of psoriasis, J. Invest. Dermatol. 68 (1) (1977) 53-60. doi:10.1111/ 1523-1747. ep12485169.

[37] A. Ikeda, N. Umeda, K. Tsuda, S. Ohta, Scanning electron microscopy of the capillary loops in the dermal papillae of the hand in primates, including man, Microsc. Res. Tech. 19 (4) (1991) 419-428. doi:10.1002/jemt.1060190404. 
[38] B. Amsden, M. F. A. Goosen, Transdermal delivery of peptide and protein drugs: an overview, AIChE Journal 41 (1995) 1972-1997. doi :https://doi. org/10.1002/aic.690410814.

[39] F. Oesch, E. Fabian, B. Oesch-Batlomowicz, C. Werner, R. Landsiedel, Drugmetabolizing enzymes in the skin of man, rat, and pig, Drug Metabolism Reviews 39 (2007) 659-98. doi:10.1080/03602530701690366.

[40] B. Al-Qallaf, D. Mori, L. Olatunji, D. B. Das, Z.Cui, Transdermal drug delivery by microneedles: Does skin metabolism matter?, International Journal of Chemical Reactor Engineering 7 (2009) 1-23. doi:10.2202/1542-6580. 1907.

[41] A. Rzhevskiy, R. Guy, Y. Anissimov, Modelling drug flux through microporated skin, J. Controlled Release 241 (2016) 194-199. doi:10.1016/j. jconrel.2016.09.029.

[42] A. Comsol, Heat Transfer Module User's Guide, AB Comsol, Stockholm, Sweden, 2012.

[43] T. Łodygowski, W. Sumelka, Limitations in application of finite element method in accoustic numerical simulation, Journal of Theoretical and Applied Mechanics 44 (4) (2006) 849-865.

[44] H. Schaefer, G. Stuttgen, Absolute concentrations of an antimycotic agent, econazole, in the human skin after local application., Arzneimittelforschung 26 (1976) 432-435.

[45] A. Davidson, B. Al-Qallaf, D. Das, Transdermal drug delivery by coated microneedles: Geometry effects on effective skin thickness and drug permeability, Chem. Eng. Res. Des. 86 (2008) 1196-1206. doi:10.1016/j . cherd. 2008.06. 002. 


\section{Appendix A}

In order to describe the concentration of the drug within the epidermal and dermal regions of the skin equations (12) and (13) will be solved. However, since we want an expression for the concentration of the solute with respect to the depth, the expressions will be rewritten to only consider depth. This means we will be assuming that the concentration of the solute will be constant across any given depth and using this assumption we find the following expressions for the change in concentration in each skin layer.

$$
\begin{gathered}
\frac{\partial C_{v e}}{\partial t}=D \frac{\partial^{2} C_{v e}}{\partial x^{2}} \\
\frac{\partial C_{d}}{\partial t}=D \frac{\partial^{2} C_{d}}{\partial x^{2}}-k_{e} C_{d}
\end{gathered}
$$

For these two equations, we have four boundary conditions that must be stated. One condition is that the flux of the drug entering the viable epidermis is constant and equal to the flux within the stratum corneum. This is a condition that has been present in each of our other models so it must be kept for this one. Another boundary condition is present at the bottom of the dermis, where the concentration of the solute will become very small and thus approach zero. Finally, there are also two boundary conditions on the boundary between the epidermis and dermis. At this boundary we will intuitively be assuming that the concentration and flux of the solutes in both compartments will be equal. A summary of these boundary conditions can be seen below:

$$
\begin{gathered}
-\left.D \frac{\partial C_{v e}}{\partial x}\right|_{x=0}=J_{s c} \\
C_{d}\left(h_{c l}\right)=K C_{v e}\left(h_{c l}\right)
\end{gathered}
$$




$$
\begin{gathered}
-\left.D \frac{\partial C_{v e}}{\partial x}\right|_{x=h_{c l}}=-\left.D \frac{\partial C_{d}}{\partial x}\right|_{x=h_{c l}} \\
\lim _{x \rightarrow \infty} C_{d}(x)=0
\end{gathered}
$$

To solve this partial differential problem, Laplace transforms can be applied to the two differential equations seen in (24) and (25). By performing Laplace transforms on these equations, we will be able to find solutions to partial differential equations. However, using these transforms does have the prerequisite of the parameters being independent of time. So therefore, we will be assuming the diffusion coefficient is constant and does not change with time. Applying the Laplace transform to the two equations for the concentration gives the following equations for the epidermal and dermal compartments:

$$
\begin{gathered}
\hat{C}_{v e}(x, s)=A \cosh \left(\sqrt{s t_{d}} \frac{x}{h_{c l}}\right)+B \sinh \left(\sqrt{s t_{d}} \frac{x}{h_{c l}}\right) \\
\hat{C}_{d}(x, s)=F \exp \left(\sqrt{\frac{s+k_{e}}{D} x}\right)+G \exp \left(-\sqrt{\frac{s+k_{e}}{D} x}\right)
\end{gathered}
$$

where $t_{d}$ is the time for diffusion and is given by $t_{d}=\frac{h_{c l}^{2}}{D}$. By using the first boundary condition stated in (26), it can be seen that $B=\frac{J_{s c}}{s \sqrt{s D}}$. Next, the fourth boundary conditions seen in (29) is applied to the dermal concentration shown in (31), such that $F=0$. Finally, when using the boundary conditions seen in (27) and (28) to equations (30) and (31), the final coefficients in each of the expression for the concentration can be found and simplify the model to:

$$
\begin{gathered}
\hat{C}_{v e}(x, s)=V \cosh \left(\sqrt{s t_{d}} \frac{x}{h_{c} l}\right)+\frac{J_{s c}}{s \sqrt{s D}} \sinh \left(\sqrt{s t_{d}} \frac{x}{h_{c l}}\right) \\
\hat{C}_{d}(x, s)=\left(W+\frac{J_{s c}}{s} \sqrt{\frac{s+k_{e}}{D}} \cosh \left(\sqrt{s t_{d}}\right)\right) \exp \left(\sqrt{\frac{s+k e}{D}\left(h_{c l}-x\right)}\right)
\end{gathered}
$$


where:

$$
V=\frac{\frac{J_{s c}}{s \sqrt{s D}}\left(\sqrt{\frac{s+k_{e}}{D}} \sinh \left(\sqrt{s t_{d}}\right)+\frac{\sqrt{s t_{d}}}{h} \cosh \left(\sqrt{s t_{d}}\right)\right)}{\frac{D \sqrt{s t_{d}}}{h} \sinh \left(\sqrt{s t_{d}}\right)+\sqrt{\left(s+k_{e}\right) D} \cosh \left(\sqrt{s t_{d}}\right)}
$$

and

$$
W=\frac{\frac{J_{s c}}{s \sqrt{s D}}\left(\sqrt{\frac{s+k_{e}}{D}} \sinh \left(\sqrt{s t_{d}}\right)+\frac{\sqrt{s t_{d}}}{h} \cosh \left(\sqrt{s t_{d}}\right)\right)}{\frac{\sqrt{s t_{d}}}{h} \sinh \left(\sqrt{s t_{d}}\right)+\sqrt{\frac{s+k_{e}}{D}} \cosh \left(\sqrt{s t_{d}}\right)}
$$

From here, a few trigonometric identities and expressions can be used to simplify this equation further. After performing this simplification, the following expressions for the concentration are obtained:

$$
\begin{gathered}
\hat{C}_{v e}(x, s)=\frac{\frac{J_{s c}}{s} \cosh \left(\sqrt{s t_{d}}\left(1-\frac{x}{h_{c l}}\right)\right)+\frac{J_{s c} \sqrt{s+k e}}{s \sqrt{s}} \sinh \left(\sqrt{s t_{d}}\left(1-\frac{x}{h_{c l}}\right)\right)}{\frac{\sqrt{s t_{d}}}{h_{c l}} \sinh \left(\sqrt{s t_{d}}\right)+\sqrt{\frac{s+k_{e}}{D}} \cosh \left(\sqrt{s t_{d}}\right)} \\
\hat{C}_{d}(x, s)=\frac{\left.\frac{J_{s c}}{s} \cosh ^{2}\left(\sqrt{s t_{d}}\right)-\sinh ^{2}\left(\sqrt{s t_{d}}\right)\right)}{\frac{D \sqrt{s t_{d}}}{h_{c l}} \sinh \left(\sqrt{s t_{d}}\right)+\sqrt{\left(s+k_{e}\right) D} \cosh \left(\sqrt{s t_{d}}\right)} \exp \left(\sqrt{\frac{s+k_{e}}{D}}\left(h_{c l}-x\right)\right)
\end{gathered}
$$

Now that we have simplified our model significantly, the steady state concentration of the drug in each layer of the skin can be found. The steady state concentration in each layer can be found by finding the limit when $s \rightarrow 0$. By finding this limit, it can be seen that $C_{v e}^{s s}(x)=\lim _{t \rightarrow \infty} C_{v e}=\lim _{s \rightarrow \infty} s \hat{C_{v e}}$. Performing this limit for the expressions for the concentration of the solute in both the dermal and viable epidermal layers of the skin seen in equations (34) and (35) produces the following steady state expressions for the concentration of the drug.

$$
\begin{gathered}
C_{v e}^{s s}(x)=\frac{J_{s c}}{\sqrt{D k_{e}}}+\frac{J_{s c} h_{c l}}{D}\left(1-\frac{x}{h_{c l}}\right) \\
C_{d}^{s s}(x)=\frac{J_{s c}}{\sqrt{D k_{e}}} \exp \left(\sqrt{\frac{k_{e}}{D}}\left(h_{c l}-x\right)\right)
\end{gathered}
$$


where $x$ is the depth within the membrane 